\title{
Teoría y tratamiento de la depresión: ¿Hacia su integración?
}

\author{
Patrick Luyten, Benedicto Lowyck \& Jozef Corveleyn
}

Universidad de Lovaina

Bélgica

Nuestra visión sobre la depresión ha cambiado de manera considerable con la investigación reciente. El Plan estratégico para la investigación de los desórdenes del carácter del Instituto Nacional de Salud Mental (INSM, 2003) es una propuesta ambiciosa orientada a crear un nuevo ímpetu en la investigación de desórdenes del carácter por un lado, y por otro a desarrollar teorías más amplias que permitan organizar estrategias de tratamiento más efectivas. Sin embargo, desde nuestro punto de vista, el plan estratégico no ha considerado algunas investigaciones recientes ni muchos problemas altamente relevantes en el ámbito de la depresión. En el presente artículo muchos puntos de divergencia importantes se precisan con respecto a una conferencia sobre la integración de teorías y estrategias de tratamiento sobre la depresión realizada el 14 de marzo del 2003 en Kortenberg (Bélgica).

depresión / vulnerabilidad biológica / terapia / interacción paciente-tratamiento

\section{Theory and treatment in depression: Towards an integration?}

Recent research has changed our view on depression in important ways. The National Institute of Mental Health (NIMH) Strategic Plan for Mood Disorders Research (NIMH, 2003) is currently the most ambitious attempt to give a new impetus to research on mood disorders, and to develop more encompassing theories that will ultimately lead to more effective treatment strategies. However, in our view, this strategic plan fails to address several important issues and recent developments in research on depression. In this article, several important points of divergence are outlined with special reference to a conference on the integration of theories and treatment strategies on depression held in Kortenberg (Belgium) on March 14, 2003.

depression / biological vulnerability / therapy / interaction treatment-patient

Traducción: Dora Herrera

Correo electrónico: Patrick.Luyten@psy.kuleuven.ac.be 


\section{INTRODUCCIÓN}

Hasta hace poco, la depresión ha sido vista como desorden prevalente pero relativamente "benigno"; incluso en ausencia de tratamiento, este desorden ha sido asociado a un buen pronóstico. La depresión fue llamada a menudo el "frío común" de la psicopatología. Sin embargo, durante las dos últimas décadas, esta visión de la depresión ha cambiado dramáticamente (Costello et al. 2002).

En primer lugar, estudios epidemiológicos recientes han mostrado que la depresión es más prevalente y debilitante que lo que se pensaba. La prevalencia de vida de la depresión mayor es estimada en aproximadamente 15\% (Blazer, Kessler, McGonagle \& Swartz, 1994) y se espera que, hacia el 2020, la depresión sea el segundo desorden más serio con respecto de las enfermedades globales (Murray \& Lopez, 1996). Además, la investigación ha aclarado progresivamente que la depresión a menudo tiene su primera aparición en la infancia o adolescencia (Costello et al., 2002), que está asociada con alta recurrencia y recaídas (Frank et al., 2002) y que tiende a ser crónica para un grupo considerable de sujetos (Kupfer \& Frank, 2001). Estimados recientes indican que el porcentaje de recaída es de 20\%-30\% durante los tres primeros años luego de la aparición del primer episodio depresivo, y de 70\%-80\% en el mismo período para sujetos que ya han tenido tres o más episodios depresivos (Segal et al., en prensa; Solomon et al., 2000). El riesgo de al menos otro episodio luego del primero es estimado en casi $90 \%$ (Kupfer \& Frank, 2001) y el número promedio de éstos es estimado en cuatro (Judd, 1997). La investigación ha mostrado también que con cada nuevo episodio, el tiempo entre dos episodios se acorta (Solomon et al., 2000) y la depresión sigue un curso crónico en aproximadamente $10 \%$ a $30 \%$ de 1 os sujetos (Verheul, 2003).

Posteriormente se ha demostrado una alta comorbilidad de los desórdenes de la depresión y la personalidad, estimando una variación entre $50 \%$ y $60 \%$ en poblaciones psiquiátricas (Klein \& Hayden, 2000; Mulder, 2002). Es ampliamente aceptado que la comorbilidad de los desórdenes de personalidad tiene un impacto negativo sobre el pronóstico y tratamiento de la depresión (Mulder, 2002).

Todo lo planteado nos orienta a un segundo dominio en el cual nuestra visión de depresión ha cambiado dramáticamente. El tratamiento psicoterapéutico y farmacológico de la depresión ha probado ser mucho más difícil y menos exitoso de lo que se esperaba. Desde 1980 hasta la actualidad, muchos investigadores y clínicos creyeron que la depresión podría ser tratada efectivamente con psicoterapias cortas (estandarizadas) tales como la terapia cognitiva conductual (TCC) o terapia interpersonal (TI) y/o tratamiento farmacológico (ver Dobson, 1989; APA, 2000).

Este optimismo ha sido seriamente moderado por un considerable cuerpo de conocimientos e investigación que demuestra que los tratamientos de 
corto plazo tienen limitaciones importantes (Hollon et al., 2002; Parker, Roy \& Eyers, 2003; Rush \& Thase, 2002; Westen \& Morrison, 2001). Por ejemplo, en lo que probablemente es el más prestigioso juicio clínico probabilístico (JCP) que se debe señalar, el Instituto Nacional de Salud Mental apoyó al programa de investigación que colaboraba en el tratamiento de la depresión (Elkin et al., 1985), sólo aproximadamente la mitad de pacientes tratados por terapia cognitivo-conductual o terapia interpersonal o medicamentos antidepresivos (Imipramine con manejo clínico) reunieron los criterios de remisión al terminar (después de 16 semanas de tratamiento). Además, los rangos de recaída fueron muy altos. En los 18 meses de seguimiento, sólo el 20\% de los pacientes no reunieron el criterio que indicaba recaída.

Westen \& Morrison (2001), a través de estudios sobre tratamientos de depresión, apoyados empíricamente, estimaron que del bien diseñado juicio clínico probabilístico sólo $50 \%$ de los pacientes deprimidos que completaron el tratamiento, mostraron mejoría. Para la muestra que intentó el tratamiento el porcentaje fue sólo del $37 \%$. Además, debido a los altos rangos de exclusión en estudios de resultados, los pacientes que tienen práctica de rutina podrían incluso mostrar menores respuestas a estos tratamientos. Por ejemplo, pacientes con depresión psicótica o peligro severo de suicidio son típicamente excluidos de estos estudios. Congruente con estas hipótesis, Westen \& Morrison (2001) encontraron que había una alta correlación entre resultado y criterio de exclusión ( $\mathrm{r}=.41)$, eso significa que cuanto más pacientes se excluyen en un estudio, mayor cantidad de pacientes han mejorado.

Adicionalmente, Parker, Roy \& Eyers (2003) han argumentado que los efectos de la terapia cognitivo-conductual, junto con la terapia interpersonal de tratamiento psicosocial de elección, de acuerdo con ciertas pautas (por ejemplo: APA, 2000), no se deben necesariamente a los factores teóricos propuestos o a técnicas terapéuticas específicas, tales como el cambio automático de pensamientos $\mathrm{y}$ actitudes disfuncionales, pues la ganancia máxima es lograda en las primeras sesiones, antes de que estas técnicas sean aplicadas. Se necesita mayor investigación respecto de los efectos de la terapia interpersonal.

Además de los efectos modestos de los tratamientos cortos, existe también una ausencia de estudios controlados sobre los efectos en el largo plazo de los tratamientos de la depresión empíricamente apoyados. Westen \& Morrison (2001), por ejemplo, pudieron identificar sólo cuatro estudios publicados en 1990, en ellos se investigaron los efectos en el largo plazo (de 12 a 18 meses) de los tratamientos de la depresión empíricamente apoyados. El porcentaje promedio de los pacientes que no mostraron recaídas después de 1218 meses fue del $36,6 \%$. Westen \& Morrison (2001) encontraron sólo un estudio con seguimiento de los datos por dos años o más. Los resultados de este estudio demostraron que sólo el 
$27 \%$ de los pacientes que estuvieron en tratamiento y el $8 \%$ de todos los pacientes inicialmente evaluados no mostraron recaídas. Westen \& Morrison (2001, p. 886) correctamente concluyeron: "Por algún standard, es difícil construir estos datas como evidencia de la hipótesis que señala que estos tratamientos muestran una eficacia genuina para el tratamiento de los desórdenes depresivos".

Como se ha mencionado, la alta comorbilidad de la depresión con otros desórdenes, como los de ansiedad, y particularmente los de personalidad, nos orienta a asunciones más modestas acerca de la efectividad del tratamiento en el corto plazo (Hollon et al., 2002).

\section{LA BÚSQUEDA DE TRATAMIENTOS}

\section{MÁS EFECTIVOS Y LA NECESIDAD DE}

\section{INTEGRACIÓN EN LA INVESTIGACIÓN}

\section{SOBRE DEPRESIÓN}

Los hallazgos de investigación discutidos previamente sugieren con claridad que es necesario realizar mayor investigación sobre la depresión, sus orígenes, los factores que influyen en su desarrollo y su tratamiento; todos estos aspectos se convierten en tareas importantes para el futuro de un científico, clínico y para una perspectiva nacional de salud mental. Como investigadores clínicos tenemos una responsabilidad importante al buscar los tratamientos apropiados para la depresión y, donde sea posible, las estrategias de prevención.

Es sorprendente que los hallazgos descritos previamente con respecto al curso recurrente y a menudo crónico de la depresión; los altos rangos de comorbilidad con desórdenes de personalidad y la progresiva constatación de las limitaciones de los tratamientos en el corto plazo, nos hayan llevado a un renovado interés de teorías y técnicas, como las psicodinámicas, que se basan en tratamientos en el largo plazo de la depresión (De Rubeis \& Cris-Cristoph, 1998; Jones \& Pulos, 1993; Kwon, 1999; Safran \& Inck, 1995; Shapiro et al., 1994); también, nos conduce a modelos que se centran en la relación existente entre personalidad y depresión (Gunderson et al., 1999; Kupfer \& Frank, 2001; Verheul, 2003).

La convergencia entre las teorías psicodinámica y cognitivo-conductual de la depresión juega un rol importante en esta evolución (Robins, 1993). La mayor parte de la investigación en este dominio se ha concentrado en la formulación psicodinámica de Sidney Blatt (Universidad de Yale, New Haven, USA) y las teorías cognitivo conductuales de Aaron T. Beck (Universidad de Pennsilvania, USA). Ambos, Blatt $(1974,1998)$ y Beck $(1983$, 1999) han propuesto dos dimensiones de la personalidad: dependencia interpersonal y perfeccionismo como factores vulnerables para la depresión. De acuerdo con Blatt y Beck, estas dos dimensiones de personalidad se encuentran asociadas con diferentes factores de desarrollo, estructuras de personalidad, estilos relacionales y de apego, interacción con eventos de vida, presentaciones clínicas y vulnerabilidades biológicas. El apoyo a estas formulaciones se basa en tres décadas de inves- 
tigación empírica (Clark \& Beck, 1999; Luyten, Corveleyn \& Blatt, 2003, en prensa).

Pese a su importancia, esta investigación se conoce muy poco, incluso entre expertos en investigación sobre depresión; esto ilustra la fragmentación existente. Distintas tradiciones teóricas y de investigación con respecto a la depresión a menudo se han desarrollado separadamente. Sin embargo, parece que es el momento exacto para articular las diferencias con el propósito de desarrollar un marco teórico de referencia más preciso con respecto a la depresión y organizar estrategias de tratamiento más efectivas que se basen en este marco integral.

La necesidad de integración ha sido recientemente reconocida de manera explícita por el Instituto Nacional de Salud Mental a través de su Plan estratégico para la investigación de los desórdenes del carácter (2003). Este plan estratégico guiará las iniciativas de investigación del instituto durante los próximos años; actualmente identifica múltiples brechas en nuestro conocimiento de los desórdenes del carácter y ha precisado estrategias de investigación que las aminorarán. Pese a que estas iniciativas merecen especial reconocimiento, nosotros tenemos la opinión de que algunos problemas clínicos de gran importancia no han sido claramente orientados por este plan estratégico; de manera específica, la visión acerca de los tratamientos psicosociales de la depresión. Esto se debe parcialmente al estado fragmentado de la investigación en este campo y a las di- ferentes tradiciones teóricas de investigación en Estados Unidos y entre éste país y otros lugares del mundo, como, por ejemplo, Europa.

Esta carencia de integración y de comunicación nos orientó a la organización de una conferencia sobre depresión con representantes de una gran variedad de campos en este ámbito de investigación. El propósito de esta conferencia no fue sólo presentar un "estado del arte" de la investigación sobre depresión; se tenía también la idea de formular caminos posibles desde una perspectiva europea de integración entre las diferentes aproximaciones sobre depresión. Adicionalmente, se consideró crucial identificar barreras que limitaran la integración entre distintas aproximaciones psicológicas y biológicas de la depresión. Tomando en consideración estos propósitos, el 14 de marzo del 2002 se llevó a cabo una conferencia de integración de las aproximaciones psicológica, biológica y psicodinámica; ésta se desarrolló en el Centro Psiquiátrico Universitario de Saint Joseph en Kortenberg (Lovaina, Bélgica). La conferencia fue organizada por el profesor doctor Jozef Corveleyn y el doctor Patrick Luyten, del departamento de psicología de la Universidad Católica de Lovaina, en Bélgica. Los organizadores invitaron a muchos expertos en la investigación sobre depresión; el principal invitado fue el profesor doctor Sidney J. Blatt, de la Universidad de Yale (New Haven, Estados Unidos), quien fue a su vez profesor visitante en la Universidad Católica de Lovaina durante ese perío- 
do. En las siguientes secciones, se discutirán distintos temas de la conferencia que son relevantes para el Plan estratégico de investigación sobre desórdenes del carácter del Instituto Nacional de Salud Mental.

\section{Cambiando la visión sobre la DEPRESIÓN}

En la conferencia de inauguración del evento, el profesor doctor Demyttenaere (Facultad de medicina de la Universidad Católica de Lovaina, Bélgica) presentó una revisión muy interesante acerca de los últimos estudios epidemiológicos sobre la depresión en el nivel internacional; se incluyó el último estudio europeo sobre la epidemiología de los desórdenes mentales del cual el doctor Demyttenaere es el coordinador belga. Su revisión mostró que la depresión es un desorden altamente prevalente también en Europa; los datos de prevalencia en este contexto eran similares a los de Estados Unidos. También se señaló, tal vez esto podría ser más importante, que la depresión no debería ser conceptualizada como un desorden aislado y discreto; al contrario, las recaídas luego del primer episodio de depresión parecerían ser la regla; por ende, con cada episodio depresivo, la probabilidad de un episodio subsecuente se incrementa. Además, Demyttenaere señaló que la depresión tiene consecuencias posteriores sobre el funcionamiento psicosocial (las 1lamadas "hipótesis cicatriz"), lo cual puede ser detectado también en un nivel neurobiológico.
De acuerdo con lo planteado, no debe sorprendernos si los episodios depresivos subsecuentes tienden a tomar un curso más autónomo y aparecen de manera menos asociada con eventos de vida discretamente negativos o estresores crónicos.

Finalmente, Demyttenaere también señaló el hecho de que la investigación epidemiológica no apoya la distinción categórica que plantea el Manual estadístico de desórdenes mentales (DSM IV, APA, 1994), en ella se distingue entre varios tipos de desórdenes depresivos (por ejemplo: depresión mayor y depresión menor). La investigación empírica ha mostrado que la depresión debe ser conceptualizada como un fenómeno continuo que va desde la ligera disforia hasta la depresión clínica clara. Nosotros opinamos que ésta es una discrepancia importante con la visión dominante americana que considera diferentes tipos de depresión; ésta visión es también compartida por el plan estratégico del Instituto Nacional de Salud Mental.

El segundo participante, profesor doctor Hermans (Departamento de Psicología de la Universidad Católica de Lovaina, Bélgica) presentó una revisión de la investigación reciente sobre depresión desde una perspectiva cognitivo-conductual. En la misma línea de la mayoría de los participantes, Hermans señaló la importancia de la investigación en torno a factores de vulnerabilidad para explicar el nacimiento del desorden depresivo y sus recaídas. El énfasis en los factores de vulnerabilidad debe, desde nuestro punto de vista, 
ocupar un rol crucial en la investigación sobre depresión en el futuro; básicamente debido a la constatación de la existencia de un alto rango de recaídas asociadas a la depresión y a las limitaciones de los tratamientos en el corto plazo. Pese a que el plan estratégico del Instituto Nacional de Salud Mental reconoce la importancia de la investigación sobre los factores de vulnerabilidad para la depresión, eso no es suficiente para brindarle a este problema la importancia que requiere y merece. En este contexto, Hermans subrayó la necesidad de investigación experimental para identificar los factores de vulnerabilidad. Indudablemente ha habido una gran dependencia de investigaciones sobre depresión basadas en cuestionarios y diseños cuasi experimentales. Hermans ilustró estos puntos con ejemplos tomados de su propia investigación acerca del rol de la memoria autobiográfica como un factor de vulnerabilidad para el nacimiento y recaídas en el desorden depresivo.

\section{Personalidad como}

\section{VULNERABILIDAD PARA LA DEPRESIÓN}

Las tres contribuciones siguientes están centradas en la convergencia entre la orientación psicodinámica de Blatt (Blatt, 1974, 2003) y la propuesta teórica cognitiva conductual de Beck en torno a la depresión (Beck, 1983; Clark \& Beck, 1999). Estas teorías conceptualizan dos dimensiones de personalidad, por ejemplo, dependencia interpersonal y perfeccionismo como factores de vulnerabilidad para formas clínicas y no clínicas de depresión. Estas dos dimensiones definen dos tipos de depresión, a saber, un tipo de depresión dependiente versus la autocrítica; con todas las posibles mixturas de depresión dependiente y autocrítica entre ellas. Además, Blatt y Beck han propuesto que la dependencia y el perfeccionismo se encuentran asociados con una vulnerabilidad específica para diferentes eventos de vida (pérdida vs. fracaso), con una presentación clínica distinta y diferente estilo relacional. También, Blatt propuso que la dependencia y el perfeccionismo tienen un origen distinto (Blatt \& Maroudas, 1992) y que los individuos dependientes y autocríticos son vulnerables a diferentes enfermedades somáticas (Blatt, Cornell \& Eshkol, 1993).

El tercer expositor en la conferencia, doctor Patrick Luyten (Departamento de Psicología de la Universidad Católica de Lovaina, Bélgica) presentó una revisión crítica de la investigación empírica de estas teorías. En su presentación señaló que, pese a que estas teorías tienen soporte teórico en diferentes aspectos, se necesita más investigación, particularmente acerca de las implicancias clínicas de estas teorías.

\section{¿LAS PERSONAS DISTINTAS REQUIEREN DIFERENTES APROXIMACIONES?}

Investigar la interacción entre el paciente y las características del tratamiento es altamente relevante para el área de investigación sobre el tratamiento de la depresión que a menudo 
se ha descuidado. Blatt (\& Felsen, 1993) y Beck (1983) han formulado perspectivas interesantes e hipótesis en este contexto. De acuerdo con Blatt y Beck, los pacientes perfeccionistas y dependientes podrían necesitar no sólo una aproximación terapéutica distinta, sino también diferente tipo de terapia (las personas distintas necesitan diferentes aproximaciones). Se espera que los pacientes dependientes sean más proclives a responder a la dimensión interpersonal y de apoyo en terapia, y por tanto hacia la propia relación terapéutica. Pese a que se espera que ellos estén listos a aceptar las interpretaciones, podría no haber mucho del contenido de éstas que sean importantes para estos pacientes, pues ellos suelen considerar con mayor énfasis las interpretaciones como evidencia de apoyo y empatía. Se espera que los pacientes perfeccionistas, por otro lado, sean más proclives a responder a los aspectos interpretativos de la psicoterapia; ello debido a su énfasis en las explicaciones intelectuales y racionales. La alianza terapéutica de los pacientes perfeccionistas es la necesidad de control y sus actitudes, de alguna manera paranoides, que tipifican su estilo relacional. Según ello, necesitan más tiempo para establecer una relación terapéutica apropiada.

De acuerdo con Blatt, además es también posible que los pacientes dependientes y perfeccionistas respondan a diferentes tipos de psicoterapia (Blatt $\&$ Felsen, 1993). Se espera que los pacientes dependientes obtengan mayor beneficio de la psicoterapia en el corto plazo; en ella los terapeutas presentan una postura más activa, directiva y de apoyo. Los pacientes perfeccionistas, por otro lado, responden mejor a psicoterapias prolongadas, orientadas hacia la comprensión súbita; ello debido a que la búsqueda de la comprensión súbita y la interpretación son centrales en este tipo de terapias. El hecho de que los terapeutas tengan una posición menos directiva y que la cantidad de sesiones no sea fija interferirá menos con la necesidad de estos sujetos por control y autonomía.

Pese a que se necesitan más estudios, el interés por investigar estas hipótesis ha producido resultados prometedores (Blatt, Shahar \& Zuroff, 2002). Por ejemplo, en el reanálisis del programa de investigación que colabora en el tratamiento de la depresión del Instituto Nacional de Salud Mental (Elkin et al., 1985), el perfeccionismo predijo resultados más limitados en todas las condiciones de tratamiento en ambas situaciones, es decir, al terminar (después de 16 semanas) y a los 18 meses (seguimiento) (Blatt et al, 1995). Tal como se esperaba, los efectos negativos del perfeccionismo sobre el resultado fue parcialmente explicado por el hecho de que los pacientes perfeccionistas mostraron menor mejoría que los otros pacientes en la alianza terapéutica (Blatt et al, 1998). El reanálisis de los datos del proyecto de investigación psicoterapéutica de Menninger, que comparó la eficacia del psicoanálisis (cinco veces por semana) y la psicoterapia psicodinámica expresiva y de apoyo (dos veces por semana), mostró 
que los pacientes autocríticos respondieron mejor al psicoanálisis que aquellos que participaron de la psicoterapia psicodinámica; se encontró lo opuesto para pacientes dependientes. Estos hallazgos son consistentes con las predicciones de Blatt (Blatt, 1992).

Los efectos de la dependencia y el perfeccionismo sobre el tratamiento farmacológico no han sido investigados de manera sistemática y nos ha conducido, en general, a resultados mixtos (Luyten, Corveleyn \& Blatt, 2003 , in press). Un hallazgo consistente fue, sin embargo, que la farmacoterapia en el corto plazo no altera de manera significativa los niveles de dependencia y perfeccionismo (Franche \& Dobson, 1992; Zuroff et al, 1999). Lo mismo se ha encontrado para los efectos de la psicoterapia en el corto plazo sobre los niveles de dependencia y perfeccionismo (Zuroff et al., 1999).

El hecho de que la psicoterapia y farmacoterapia en el corto plazo no haya logrado generar cambios significativos en la personalidad, podría explicar por qué los efectos de estos tratamientos en el corto plazo son limitados y por qué la tasa de recaídas, luego de terminar los tratamientos, es tan alta.

Si la personalidad, tal como lo sugiere la investigación, es realmente un factor de vulnerabilidad para el inicio o las recaídas de la depresión, entonces, la alta tasa de recaídas no debería sorprendernos. Esto podría también explicar parcialmente por qué algunos pacientes responden a los tratamientos en el corto plazo, pero recaen rápidamente luego de culminar la terapia. Es posible que algu- nos pacientes -particularmente los dependientes- instalen en ese período corto (los cuales son a menudo de gran apoyo) una nueva relación de dependencia con su terapeuta, el cual lo lleva a una rápida mejoría de sus síntomas. Esta información es consistente con el hecho de que la mejoría en terapias cortas a menudo aparece en el período de introducción de las técnicas formales (por ejemplo, en las primeras sesiones) (Parker et al., 2003). Sin embargo, al terminar la terapia, los beneficios de esta dependencia y esperanzadora relación o "cura de transferencia" culminan.

La creciente evidencia de la terapia de continuación y mantenimiento en el tratamiento de la depresión (Rush \& Thase, 2002) pueden, desde nuestra perspectiva, ser parcialmente explicados a través de líneas similares. La terapia de continuación o mantenimiento hará posible, para los pacientes dependientes, continuar o prolongar su relación de dependencia con su terapeuta; una relación caracterizada por la orientación, cuidado y apoyo. Beck (1983) ha argumentado que los pacientes dependientes son por lo general muy optimistas acerca del tratamiento, con ello logran un significativo, pero a menudo temporal, alivio de los síntomas. En suma, nosotros señalamos que no sólo los pacientes perfeccionistas, también los dependientes, a menudo no necesitan un tratamiento agudo seguido por tratamientos de mantenimiento o continuidad; no obstante un tratamiento prolongado, tomando en consideración las vulnerabilidades, tales como dependencia y autocriticismo, podría ser trabajado. 
¿QUÉ FUNCIONA EN PSICOTERAPIA?

\section{LA IMPORTANCIA DE LAS}

\section{CARACTERÍSTICAS DE LOS}

\section{PACIENTES Y TERAPEUTAS}

Tal como se enfatizó por varios participantes en nuestra conferencia, los hallazgos relacionados con la influencia de la personalidad sobre los resultados son congruentes con los hallazgos generales en el dominio de la investigación psicoterapéutica. Se ha considerado, cada vez con mayor fuerza, que la terapia prolongada es necesaria para muchos pacientes (Kupfer \& Frank, 2001; Westen \& Morrison, 2001) y que las características de los pacientes y los terapeutas son predictores más importantes de los resultados que las técnicas terapéuticas específicas (Wampold, 1997, 2002). Al respecto, Blatt, Sanislow et al. (1998) encontraron que los terapeutas efectivos, independientemente del tipo de tratamiento que brindaron, fueron orientados más psicológica que biológicamente, y usaron más psicoterapia que tratamiento médico para la depresión. Este estudio mostró también que los terapeutas más efectivos creyeron que se necesitaba mayor tiempo para obtener un cambio. Finalmente, los terapeutas efectivos mostraron menor variabilidad en su resultado; parecía que ellos tenían la posibilidad de trabajar de manera más efectiva con la mayoría de sus pacientes. Estos hallazgos proveen una buena ilustración de la importancia de las variables del terapeuta al margen de la clase de tratamiento que ofrecen.

Desde nuestro punto de vista, esto significa que la investigación futura no debe centrarse únicamente en estudiar la eficacia de varias formas de psicoterapia, sino, y tal vez de manera muy urgente, en estudios detallados acerca del rol del paciente y de las características del terapeuta que pueden influir en los resultados. Sólo la investigación del proceso puede señalarnos cómo un tratamiento particular funciona. Además, podría también identificar los factores del paciente que influyen en el resultado y de esta manera podría orientarnos a decisiones más racionales de tratamientos.

A menudo los tratamientos de la depresión apoyados en datos empíricos son vistos como apropiados para todos los pacientes (APA, 2000). O mejor dicho, no se sabe qué tratamiento sustentado en datos empíricos funciona mejor para algunos pacientes. Esto significa que, hasta la fecha, la mayoría de las decisiones sobre tratamiento farmacológico o psicosocial se basan en preferencias afectivas de los terapeutas o pacientes involucrados.

\section{LA NECESIDAD DE UN MARCO DE}

\section{REFERENCIA INTEGRAL}

En su presentación de la conferencia sobre depresión en Kortenberg, Sidney Blatt se centró principalmente en sus recientes elaboraciones sobre depresión y sobre psicopatología en general. Se enfatizó acerca de su visión sobre la depresión que, así como la de Beck, es parte de una teoría más general sobre el desarrollo de la personalidad normal y patológica (Blatt \& Shichman, 1983; Beck, 1999). Al igual que otros confe- 
rencistas, Blatt no ve la depresión como una entidad aislada, sino como resultado de cierta vulnerabilidad que puede ser el origen de una distorsión o exageración de tareas de desarrollo normales.

De acuerdo con Blatt, el desarrollo de la personalidad puede ser conceptualizado como una interacción dialéctica entre dos líneas de desarrollo, a saber

- una línea analítica o relacionada que en términos de desarrollo normal nos orienta a la progresiva madurez y relaciones interpersonales diferenciadas y satisfactorias.

- una línea introyectiva de autodefinición, la cual normalmente conduce hacia la estabilidad, esencialmente positiva autodefinición y sentido del yo.

Basándose en esta visión, la psicopatología puede ser conceptualizada como una exageración o distorsión de una línea de desarrollo y la negación defensiva de la otra. Esta visión nos permite establecer una distinción entre dos agrupaciones psicopatológicas, a saber, la analítica y la introyectiva. En cada grupo, Blatt distinguió muchos síntomas (eje I) y desórdenes de personalidad (eje II) sobre tres niveles estructurales de psicopatología, a saber, el psicótico, el borderline y el neurótico. En el grupo analítico, los problemas y los conflictos con respecto a las relaciones son los predominantes, por ejemplo, la intimidad, el cuidado y la sexualidad (pacientes histéricos e histriónicos), mientras que en los grupos introyectivos, los problemas y conflictos relacionados con la autonomía, el poder y la agresión son los predominantes (paranoia y pacientes obsesivocompulsivos).

Esta visión nos abre muchas perspectivas interesantes para la integración entre varias aproximaciones psicológicas y biológicas sobre la depresión. En realidad, la investigación ha mostrado que los procesos biológicos, sociales y psicológicos están interrelacionados en el desarrollo de la personalidad normal y patológica. La investigación sobre la depresión podría por tanto beneficiarse e insertarse en un marco de referencia teórico vinculado al desarrollo de la personalidad normal y patológica.

La necesidad de este marco de referencia integral fue también subrayada por el doctor Maurits Nijs (Sint-Radboud Hospital, Nijmege, Países Bajos) en su presentación. Él señaló, por ejemplo, que un diagnóstico de depresión mayor de acuerdo con el criterio del DSM IV no tiene valor predictivo, y la respuesta sobre psicoterapia o farmacoterapia. La investigación sobre la efectividad de la psicoterapia y la farmacoterapia, y especialmente la combinación de ambas, es difícil debido a una pérdida de teorías integrales. Por ejemplo, no hay una teoría sólida que ofrezca una propuesta teórica racional para combinar la psicoterapia y la farmacoterapia.

Los profesores, doctores Meurs y Cluckers, y la doctora Vliegen (Departamento de Psicología de la Universidad Católica de Lovaina, Bélgica) enfatizaron en la necesidad de teorías integrales, especialmente respecto del desarrollo de la depresión. La necesi- 
dad de teorías vinculadas a los orígenes del desarrollo de la depresión es urgente debido a que estudios recientes han mostrado que la depresión tiene a menudo sus orígenes en la infancia o la adolescencia. Mientras muchos dudan si los niños pueden experimentar depresión, la pregunta es cuántos adultos experimentan su primera depresión durante la adultez (Costello et al., 2002).

Meurs, Vliegen y Cluckers indicaron la importancia de una perspectiva psicodinámicamente orientada con respecto al desarrollo psicopatológico, la cual, en línea con otros conferencistas, no considere la depresión como una enfermedad aislada sino como un fenómeno más o menos "normal" que puede tomar la forma patológica o normal, dependiendo de la historia particular y los factores situacionales asociados.

\section{Conclusiones}

La investigación sobre depresión está indudablemente ingresando a una nueva era. En la conferencia sobre depresión en Kortenberg, Bélgica, se señaló que hay una tendencia hacia la integración de varias aproximaciones teóricas sobre la depresión. Esta propuesta se debe parcialmente al cambio de visión con respecto a la depresión que ha sido elaborado durante la última década. La depresión es un desorden prevalente y recurrente; anteriormente no se pensaba así. Los tratamientos en el corto plazo han mostrado ser menos efectivos que lo que se esperaba. Estos hechos son actualmente reconocidos y los investigadores y clínicos de diferentes partes del mundo se han enfrentado a la tarea de desarrollar nuevas teorías y formas de tratamiento. El plan estratégico para investigar los desórdenes del carácter que propone el Instituto Nacional de Salud Mental puede ser considerado como un ambicioso intento de avanzar en el conocimiento de la depresión y de realzar nuestras posibilidades de tratamiento.

Los clínicos e investigadores tienen una opinión diferente sobre las rutas que hay que seguir al construir las nuevas teorías y tratamientos. Las diferentes opiniones derivan en parte de diferentes tradiciones de investigación en Estados Unidos y entre este país y otras partes del mundo. En la conferencia sobre depresión en Kortenberg, se formularon por lo menos cuatro puntos importantes de divergencia con el plan estratégico. Es difícil discernir si estas diferencias se originan de diferentes tradiciones de investigación. Lo importante es que ellas existen y que tendrán una influencia profunda en la futura investigación sobre depresión.

Primero, si el plan estratégico del Instituto Nacional de Salud Mental no cuestiona la aproximación del DSM IV respecto de la clasificación categorial de los desórdenes del carácter; muchos participantes en la conferencia elaboraron esta pregunta y presentaron un argumento alternativo mediante el cual la depresión debe ser situada sobre un continuum, que va desde los síntomas de disforia leve hacia un desorden severo que inhabilita. A pesar de la vasta canti- 
dad de investigación (Clark \& Beck, 1999) la pregunta de si la distinción cuantitativa (y no la cualitativa) debe hacerse entre las diferentes formas de depresión aún continúa sin respuesta. Nosotros señalamos que este es un aspecto que merece ser priorizado en investigaciones futuras sobre depresión.

En segundo lugar, se remarcó la necesidad de investigar sobre factores de vulnerabilidad que influyen en el origen y recaídas de la depresión. Esto fue enfatizado con mayor fuerza en el plan estratégico. Respecto de este punto, hubo consenso en señalar que la investigación, especialmente en torno al tratamiento, en el pasado se centró demasiado en los síntomas y en la reducción de los síntomas a expensas de un funcionamiento psicosocial más amplio, sobre todo desde la perspectiva de desarrollo (Costello et al., 2002), el énfasis es principalmente planteado en la vulnerabilidad biológica y no en los factores psicosociales, tales como la personalidad o bajo soporte social.

Tercero, la importancia de la investigación de proceso, investigando los mediadores y moderadores del cambio en psicoterapia; este argumento se enfatizó más en esta conferencia que en el plan estratégico. Adicionalmente, mientras éste desea restringir la investigación de proceso hacia diseños de juicios clínicos; en la conferencia hubo más flexibilidad y apertura hacia otros; las estrategias de investigación alternativa representan diseños para investigar los procesos implicados en psicoterapia.

Finalmente, varios conferencistas indicaron la importancia de la psicote- rapia prolongada para muchos pacientes deprimidos; de esa manera resaltaron la investigación psicoterapéutica en el largo plazo. Pese a que en el plan estratégico del Instituto Nacional de Salud Mental se consideró la psicoterapia prolongada, éste es más optimista respecto de la eficacia y posibilidades de las psicoterapias en el corto plazo que están disponibles. Ese optimismo no puede justificarse a la luz de la investigación.

Adicionalmente, desde que el plan estratégico del Instituto Nacional de Salud Mental sólo reconoce algunos juicios clínicos al azar como evidencia válida para determinar la eficacia de las estrategias de tratamiento, parece excluirse de facto la investigación sobre el real rol de las psicoterapias prolongadas para la depresión. Tal como se sabe, para los juicios clínicos al azar es casi imposible investigar la eficacia de terapias en el largo plazo (Kendall, Hollmbeck \& Verduin, 2003). Por ejemplo, es éticamente inapropiado referir pacientes para una terapia prolongada a una lista de espera.

A la luz de estas diferencias, el campo de la investigación en depresión indudablemente se orienta a ser un continuum de desarrollo y discusión en el futuro próximo. Si estas diferencias y similitudes nos orientarán hacia teorías más integradoras y a tratamientos más efectivos, será constatado únicamente a través del tiempo. 


\section{REFERENCIAS}

American Psychiatric Association (1994). Diagnostic and Statistical Manual of Mental Disorders (4th. ed). DSM-IV. Washington D.C.: American Psychiatric Association.

American Psychiatric Association (2000). Practice guidelines for the treatment of patients with major depressive disorder (revision). American Journal of Psychiatry, 157 (suppl), 1-45.

Beck, A.T. (1983). Cognitive therapy of depression: New perspectives. En: P.J. Clayton \& J.E. Barrett (Eds.). Treatment of depression: Old controversies and new approaches (pp. 265290). New York: Raven Press.

Beck, A.T. (1999). Cognitive aspects of personality disorders and their relation to syndromal disorders: A psychoevolutionary approach. En: C.R. Cloninger (Ed.). Personality and psychopathology (pp.411-429). Washington DC/Londres: American Psychiatric Press.

Blatt, S. (1974). Levels of object representation in anaclitic and introjective depression. The Psychoanalytic Study of the Child, 29, 107-157.

Blatt, S.J (1992). The differential effect of psychotherapy and psychoanalysis with anaclitic and introjective patients: The Menninger psychotherapy research project revisited. Journal of the American Psychoanalytic Association, 40, 691-724.

Blatt, S.J. (1998). Contributions of psychoanalysis to the understanding and treatment of depression. Journal of the American Psychoanalytic Association, 46, 722-752.
Blatt, S.J., Cornell, C.E. \& Eshkol, E. (1993). Personality style, differential vulnerability and clinical course in immunological and cardiovascular disease. Clinical Psychology Review, 13, 421-450.

Blatt, S.J. \& Felsen, I. (1993). Different kinds of folks may need different kinds of strokes: The effect of patients' characteristics on therapeutic process and outcome. Psychotherapy Research, 3, 245-259.

Blatt, S.J. \& Maroudas, C. (1992). Convergence among psychoanalytic and cognitive-behavioral theories of depression. Psychoanalytic Psychology, 9, 157-190.

Blatt, S.J., Quinlan, D.M., Pilkonis, P.A. \& Shea, M.T. (1995). Impact of perfectionism and need for approval on the brief treatment of depression: The National Institute of Mental Health Treatment of Depression Collaborative Research Program revisited. Journal of Consulting and Clinical Psychology, 63, 125-132.

Blatt, S.J., Shahar, G. \& Zuroff, D.C. (2002). Anaclitic/Sociotropic and Introjective/Autonomous dimensions. En: J C. Norcross (Ed.). Psychotherapy relationships that work. Therapist contributions and responsiveness to patients (pp. 315-333). Oxford: Oxford University Press.

Blatt, S.J. \& Shichman, S. (1983). Two primary configurations of psychopathology. Psychoanalysis and Contemporary Thought, 6, 187-254.

Blatt, S.J., Zuroff, D. C., Bondi, C.M., Sanislow, C.A. \& Pilkonis, P.A. (1998). When and how perfectionism impedes the brief treatment of depression: 
Further analyses of The National Institute of Mental Health Treatment of Depression Collaborative Research Program. Journal of Consulting and Clinical Psychology, 6, 423-428.

Blazer, D.G., Kessler, R.C., McGonagle, K.A. \& Swartz, M. S. (1994). The prevalence and distribution of major depression in a national community sample: The National Comorbidity Survey. American Journal of Psychiatry, 151, 979-986.

Clark, D.A. \& Beck, A.T. (1999). Scientific foundations of cognitive theory and therapy of depression. Nueva York: John Wiley \& Sons.

Costello, E.J., Pine, D.S., Hammen, C., March, J.S., Plotsky, P.M., Weissman, M.M., Biederman, J., Goldsmith, H.H., Kaufman, J., Lewinsohn, P.M., Hellander, M., Hoagwood, K., Koretz, D.S., Nelson, C.A. \& Leckman, J.F. (2002). Development and natural history of mood disorders. Biological Psychiatry, 52, 529-542.

DeRubeis, R.J. \& Crits-Christoph, P. (1998). Empirically supported individual and group psychological treatments for adult mental disorders. Journal of Consulting and Clinical Psychology, 66, 37-52.

Dobson, K.S. (1989). A meta-analysis of the efficacy of cognitive therapy for depression. Journal of Consulting and Clinical Psychology, 57, 414-419.

Elkin, I., Parloff, M.B., Hadley, S. W. \& Autry, J.H. (1985). NIMH Treatment of Depression Collaborative Research Program. Archives of General Psychiatry, 42, 305-316.
Franche, R.L. \& Dobson, K. (1992). Selfcriticism and interpersonal dependency as vulnerability factors to depression. Cognitive Therapy and Research, 16 (4), 419-435.

Gunderson, J.G., Triebwasser, J., Philips, K.A. \& Sullivan, C.N. (1999). Personality and vulnerability to affective disorders. En: C.R. Cloninger (Ed.). Personality and psychopathology (pp. 3-32). Washington DC/Londres: American Psychiatric Press.

Hollon, S.D., Munoz, R.F., Barlow, D.H., Beardslee, W. R., Bell, C.C., Bernal, G., Clarke, G.N., Franciosi, L P., Kazdin, A.E., Kohn, L., Linehan, M.M., Markowitz, J.C., Miklowitz, D.J., Persons, J.B., Niederehe, G. \& Sommers, D. (2002). Psychosocial intervention development for the prevention and treatment of depression: Promoting innovation and increasing access. Biological Psychiatry, 52, 610630 .

Jones, E.E., Pulos, S.M. (1993). Comparing the process of psychodynamic and cognitive-behavioral therapies. Journal of Consulting and Clinical Psychology, 61, 306-316.

Judd, L.J. (1997). The clinical course of unipolar major depressive disorders. Archives of General Psychiatry, 54, 989-991.

Kendall, P.C., Holmbeck, G. \& Verduin, T. (2003). Methodology, design, and evaluation in psychotherapy research. En: M.J. Lambert (Ed.). Bergin and Garfield's handbook of psychotherapy and behavior change ( $5^{\text {th }}$. ed.). (pp. 1643). Nueva York: John Wiley \& Sons.

Kupfer, D.J. \& Frank, E. (2001). The interaction of drug- and psychotherapy in 
the long-term treatment of depression. Journal of Affective Disorders, 62, 131137.

Kwon, P. (1999). Attributional style and psychodynamic defense mechanisms: Toward an integrative model of depression. Journal of Personality, 67, 645658.

Luyten, P., Corveleyn, J. \& Blatt, S.J. (2003, in press). The convergence among psychodynamic and cognitivebehavioral theories of depression: A critical overview of empirical research. En: S.J. Blatt, J. Corveleyn \& P. Luyten (Eds.). Theory and treatment of depression: Towards integration? Leuven: Leuven University Press.

Mulder, R.T. (2002). Personality pathology and treatment outcome in major depression: A review. American Journal of Psychiatry, 159, 359-371.

Murray, C.J. L. \& Lopez, A.D. (Eds.) (1996). The global burden of disease: A comprehensive assessment of mortality and disability from diseases, injuries and risk factors in 1990 and projected to 2020. Cambridge MA: Harvard University Press.

National Institute of Mental Health (2003). Breaking ground, breaking through: The Strategic Plan for Mood Disorders Research.

Parker, G., Roy, K. \& Eyers, K. (2003). Cognitive behavior therapy for depression? Choose horses for courses. American Journal of Psychiatry, 160, 825-834.

Robins, C.J. (1993). Implications of research in the psychopathology of depression for psychotherapy integration. Journal of Psychotherapy Integration, 3, 313-330.
Rush, A.J. \& Thase, M.E. (2002). Psychotherapies for depressive disorders: A review. En: M. Maj \& N. Sartorius (Eds.). Depressive disorders, ( ${ }^{\text {nd }}$. ed). (pp.161-206). Chichester: Wiley.

Safran, J.D. \& Inck, T.A. (1995). Psychotherapy integration: Implications for the treatment of depression. En: E.E. Beckham \& W.R. Leber (Eds.). Handbook of depression (2 ${ }^{\text {nd }}$. ed.). (pp. 425-434). Nueva York/Londres: The Guilford Press.

Segal, Z.V., Pearson, J. \& Thase, M.E. (2003, in press). Challenges in preventing relapse in major depression. Report of a National Institute of Mental Health Workshop on state of the science of relapse prevention in major depression. Journal of Affective Disorders.

Shapiro, D.A., Barkham, M., Rees, A., Hardy, G.E., Reynolds, S. \& Startup, M. (1994). Effects of treatment duration and severity of depression on the effectiveness of cognitive-behavioral and psychodynamic interpersonal psychotherapy. Journal of Consulting and Clinical Psychology, 62, 522-534.

Shea, M.T., Elkin, I., Imber, S.D., Sotsky, S.M., Watkins, J.T., Collins, J.F., Pilkonis, P.A., Beckham, E., Glass, D. R. \& Dolan, R.T. (1992). Course of depressive symptoms over follow-up. Findings from the NIMH treatment of depression collaborative research program. Archives of General Psychiatry, 49, 782-787.

Solomon, D.A., Keller, M.B., Leon, A.C., Mueller, T.I., Lavori, P.W., Shea, M.T., Coryell, W., Warshaw, M., Turvey, C., Maser, J.D. \& Endicott, J. (2000). Multiple recurrences of major depressi- 
ve disorder. American Journal of Psychiatry, 157, 229-233.

Verheul, R. (2003). Chronische depressie en persoonlijkheidsstoornis. Overeenkomsten, onderscheid en consequenties [Chronic depression and personality disorders. Similarities, differences, and consequences]. En: M.B. J. Blom, J. Spijker \& R. Van Dyck (Eds.). Behandelingsstrategieën bij chronische depressie en dysthymie (pp. 27-44). Houten/Mechelen: Bohn Stafleu Van Loghum.

Wampold, B.E. (1997). Methodological problems in identifying efficacious psychotherapies. Psychotherapy Research, 7, 21-43.

Wampold, B.E. (2002). Indications and planning of psychotherapies: "Much ado about nothing"? En: M. Maj \& N. Sartorius (Eds.). Depressive disorders, ( $2^{\text {nd }}$. ed.). (pp. 209-211). Chichester: Wiley.

Westen, D. \& Morrison, K. (2001). A multidimensional meta-analysis of treatments for depression, panic, and generalized anxiety disorder: An empirical examination of the status of empirically supported therapies. Journal of Consulting and Clinical Psychology, 69, 875-899.

Zuroff, D.C., Blatt, S.J., Sanislow III, C. A., Bondi, C.M. \& Pilkonis, P.A. (1999). Vulnerability to depression: Reexamining state dependence and relative stability. Journal of Abnormal Psychology, 108, 76-89. 